\title{
ASPECTOS PRIORIZADOS EN LOS PLANES DE MEJORA DE LAS CARRERAS ACREDITADAS Y FOMENTO DE UNA CULTURA DE EVALUACIÓN EN LA UNIVERSIDAD DE COSTA RICA
}

PRIORITIES FAVORED BY ACCREDITED UNIVERSITY MAJORS IN THEIR SELFIMPROVEMENT PLANS AND PROMOTION OF A SELF-EVALUATION CULTURE IN THE UNIVERSITY OF COSTA RICA

Volumen 11, Número 2

pp. $1-24$

Este número se publicó el 30 de agosto de 2011

\author{
Elsiana Guido Guido \\ María Lourdes Calderón Laguna \\ Eugenia Gallardo Allen
}

La revista está indexada en los directorios:

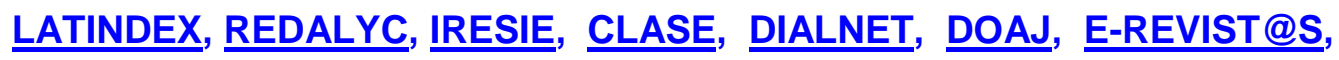

La revista está incluida en los sitios:

REDIE, RINACE, OEI, MAESTROTECA, PREAL, HUASCARAN, CLASCO 


\title{
ASPECTOS PRIORIZADOS EN LOS PLANES DE MEJORA DE LAS CARRERAS ACREDITADAS Y FOMENTO DE UNA CULTURA DE EVALUACIÓN EN LA UNIVERSIDAD DE COSTA RICA \\ PRIORITIES FAVORED BY ACCREDITED UNIVERSITY MAJORS IN THEIR SELF- IMPROVEMENT PLANS AND PROMOTION OF A SELF-EVALUATION CULTURE IN THE UNIVERSITY OF COSTA RICA
}

\author{
Elsiana Guido Guido ${ }^{1}$ \\ María Lourdes Calderón Laguna² \\ Eugenia Gallardo Allen ${ }^{3}$
}

\begin{abstract}
Resumen: El artículo describe y analiza dieciséis planes de mejora realizados por las carreras de la Universidad de Costa Rica (UCR), que fueron acreditadas por el Sistema Nacional de Acreditación (SINAES) entre los años del 2001 al 2010. Se ofrece un panorama de cuáles son las principales debilidades y acciones que han priorizado esas carreras para mejorar la calidad de sus ofertas académicas. Asimismo, se analiza la contribución los procesos de autoevaluación a la creación de una cultura de la evaluación en la UCR. Los hallazgos obtenidos de este estudio se presentan según los componentes valorados por el SINAES: currículum, administración de la carrera o programa, personal académico, estudiantes, infraestructura y equipamiento, impacto y pertinencia de la carrera. Se encontraron dos tipos de acciones de mejora, la primera de ellas relacionada con aspectos propios de la carrera y la otra que corresponde a la institución. Las categorías que más reportaron las unidades académicas fueron las que contenían acciones de mejora relacionadas con: la planta física, la actualización curricular, con la formación y reclutamiento de personal docente.
\end{abstract}

Palabras clave: AUTOEVALUACIÓN, ACREDITACIÓN UNIVERSITARIA, CULTURA DE EVALUACIÓN, UNIVERSIDAD DE COSTA RICA

\begin{abstract}
This article describes and analyzes sixteen self improvement plans conducted by academic departments in the University of Costa Rica (UCR) after their self evaluations. The academic programs or majors of these units have all been accredited by the National Accreditation System (SINAES) between 2001 and 2010. It overviews the priorities listed by academic units to improve the quality of their academic services, as well as the extent to which these processes contribute to the development of an evaluation culture in the UCR. Results are organized according to evaluation criteria listed by SINAES: curriculum, management of the academic program, faculty, students, infrastructure and equipment, impact and pertinence of each academic program or major. We found two types of improvement actions, the first of which related to specific aspects of the academic departments and the other for the institution. The categories that most academic departments reported, which contained measures, related to improvement: improving the physical plant, modernization of curricula and the training and recruitment of teachers.
\end{abstract}

Key words: SELF EVALUATION, UNIVERSITY ACCREDITATION, EVALUATION CULTURE, UNIVERSITY OF COSTA RICA

\footnotetext{
${ }^{1}$ Politóloga y Administradora de programas educativos no formales. Universidad de Costa Rica. Investigadora del Departamento de Investigación y Evaluación Académica (DIEA) del Centro de Evaluación Académica (CEA) de la Universidad de Costa Rica. Dirección electrónica: elsiana.guido@ucr.ac.cr

2 Psicóloga, Licenciada en Psicología, Universidad de Costa Rica, Investigadora del Departamento de Investigación y Evaluación Académica (DIEA) del Centro de Evaluación Académica (CEA), Universidad de Costa Rica. Dirección electrónica: maria.calderon@ucr.ac.cr

${ }^{3}$ Estadística, Magister Scientae en Estadística, Universidad de Costa Rica, Investigadora del Departamento de Investigación y Evaluación Académica (DIEA) del Centro de Evaluación Académica. Dirección electrónica: eugenia.gallardo@ucr.ac.cr
}

Artículo recibido: 16 de mayo, 2011

Aprobado: 18 de aaosto. 2011 


\section{Introducción}

El siguiente artículo tiene como fin describir y analizar el contenido de dieciséis planes de mejora realizados del 2001 al 2010 por carreras de la Universidad de Costa Rica (UCR), que fueron acreditadas por el Sistema Nacional de Acreditación de la Educación Superior (SINAES).

Cada plan de mejora fue analizado de acuerdo con los componentes que solicita la agencia, a saber: currículum, administración de la carrera o programa, personal académico, estudiantes, infraestructura y equipamiento, impacto y pertinencia de la carrera o programa. La información resultante se agrupó en categorías de análisis que engloban las principales acciones contenidas en los planes.

Usualmente, se presentan, en los planes de mejora, además de la debilidad encontrada, las causas, los objetivos, las metas, las acciones, los responsables, los tiempos dispuestos para superarlas, los resultados esperados, los plazos, los recursos y los indicadores de éxito. Para la realización de esta investigación se tomaron únicamente los objetivos señalados por las carreras acreditadas, ya que a partir de ahí se derivan las acciones concretas que conforman el plan de mejora.

En este artículo también interesa analizar de qué manera los procesos de evaluación, que subyacen a la obtención de la acreditación de las carreras, están contribuyendo con la creación de una cultura de la evaluación en la Universidad de Costa Rica.

\section{Autoevaluación, planes de mejora y cultura de evaluación}

En los últimos años, se ha incrementado a nivel mundial la práctica de realizar procesos de autoevaluación por parte de las carreras universitarias, con el propósito de certificar la calidad de la educación superior. Una evidencia de este hecho es el aumento de agencias inscritas en la Red Internacional de Agencias para la Garantía de la Calidad de la Educación Superior (INQAAHE), que pasó de 8 organizaciones miembros en 1991 a 200 en el 2011 (INQAAHE, 2011).

En el caso de Costa Rica, el Sistema Nacional de Acreditación (SINAES) fue creado en el año 1999 con la misión de "promover el mejoramiento permanente de la calidad de la educación superior” (SINAES, 2009, p. 7). Destaca, como uno de sus objetivos principales, certificar la calidad de las carreras impartidas en las instituciones de educación superior. La Universidad de Costa Rica es una de las instituciones adherente al SINAES, por lo que 
algunas de sus carreras han realizado procesos de acreditación bajo los criterios y los estándares de esta agencia.

Para obtener la acreditación, la agencia solicita una serie de insumos, a saber: el informe de autoevaluación realizado por la carrera, el informe de evaluación externa realizado por los pares evaluadores seleccionados, el plan o compromiso de mejoramiento que presenta la carrera como producto de su proceso de autoevaluación, informes y recomendaciones técnicas especializadas y otra información que pueda ser obtenida en el proceso.

Como ya se mencionó, para este artículo el análisis se centró en los compromisos o planes de mejoramiento elaborados por las carreras de la Universidad de Costa Rica que han sido acreditadas por el SINAES, y que para su proceso de autoevaluación utilizaron el Manual de acreditación, de la convocatoria del año 2000.

La autoevaluación es una evaluación realizada por la carrera de acuerdo con los criterios y los estándares brindados por la agencia de acreditación y da cuenta del estado actual de la calidad de la misma. Para el SINAES, la autoevaluación es y debe contemplar las siguientes características:

Es un proceso previo a la acreditación que debe realizar la carrera o programa que tiene intención de acreditarse. Debe tener características que den fe de la formalidad y rigor técnico con que se haya realizado y la información obtenida debe ser válida, confiable y verificable. (...) Es un proceso participativo de revisión sistemática del quehacer de la carrera o programa, organizado por un equipo de trabajo comprometido y calificado. (SINAES, 2000, p. 15)

Por su parte, y según la agencia acreditadora, el plan o compromiso de mejora de una carrera plantea el desarrollo de las acciones que, a criterio de las poblaciones consultadas en el proceso de autoevaluación, influyen, o pueden influir, de manera directa en el mantenimiento o en la mejora de los niveles de calidad.

La construcción del plan de mejora requiere de una serie de condiciones para un desarrollo óptimo. De acuerdo con la agencia SINAES, es importante que este plan sea construido con un sentido de realidad, donde se toman en cuenta los costos, el calendario y los recursos. Es también importante la viabilidad política, la técnica, la económica y la legal por parte de la institución. Además, es trascendental contar con el compromiso de todos los 
actores y las autoridades universitarias, y debe estar integrado en el plan operativo anual y en el presupuesto de la carrera.

El plan de mejora de una carrera plantea, entonces, el desarrollo de las acciones que, a criterio de las poblaciones consultadas en el proceso de autoevaluación, influyen, o pueden influir, de manera directa en el mantenimiento o en la mejora de los niveles de calidad (SINAES, 2000). De esta forma, se concibe al plan de mejora como el resultado de la interacción de fuerzas, de actores y de autoridades universitarias, que reflejan el acuerdo o negociación de todas las partes implicadas en el proceso de autoevaluación.

Es importante, en este punto, recalcar el concepto de calidad utilizado por SINAES, que se refiere al cumplimiento por parte de la carrera de los criterios establecidos por la agencia, evaluados en sus diferentes etapas, para obtener el certificado de acreditación (La Gaceta, 2002).

Como se mencionó, las carreras seleccionan y priorizan los aspectos que deben ser mejorados. Estos son incorporados a un plan de mejoramiento, que se puede visualizar metafóricamente como un vehículo, que inicia su recorrido en un tiempo t de su plan de acción $y$, al finalizar el recorrido, pasa a un momento $t+1$, donde las debilidades deben tender a ser superadas y las fortalezas vigorizadas.

Un concepto interesante mencionado por Bolseguí y Fuguet (2006) es el de cultura de evaluación, donde la evaluación se percibe como una actividad permanente e incorporada a los procesos institucionales. Se fomenta la participación de todos los sectores, para que con responsabilidad contribuyan al desarrollo institucional y al alcance de la calidad y la excelencia académica. Es así como el establecimiento de una cultura de evaluación promueve una reflexión integral y sistemática, sobre las condiciones, los procesos, los efectos de los programas y las actividades. Esta reflexión se vuelve natural en una relación de tensión, control, auto-reflexión y acción (Teichler, 2007).

Según lo anterior, cuando se habla del fomento de una cultura de evaluación, es necesario generar mecanismos y sistemas de evaluación, establecer procesos de evaluación internos promoviendo la autoevaluación como ejercicio diario, propiciar la participación y la negociación de los actores, y generar, en contexto, la promoción de valores para la convivencia social (Bolseguí y Fuguet, 2006).

Para el caso particular de las carreras estudiadas en este artículo existen una serie de interrogantes con respecto a este fomento de la cultura de la evaluación planteado por 
Bolseguí y Fuget, por ejemplo, en qué medida la UCR crea mecanismos para que los procesos de evaluación llevados a cabo con fines de acreditación sean permanentes y cotidianos; en ese sentido, existe una participación activa y reflexiva de los diferentes actores vinculados en el quehacer de la carrera, donde cada actor aportará sus juicios a la construcción de estrategias para implementarlos y, la siguiente pregunta es qué tanto estas prácticas se incorporan como actividades periódicas asociadas a la transformación para mejoramiento.

Seguidamente se explica el procedimiento metodológico seguido y se muestran los hallazgos encontrado en el análisis.

\section{Procedimiento metodológico}

En el presente artículo se analizaron dieciséis compromisos o planes de mejora elaborados por las carreras de la Universidad de Costa Rica, acreditadas por el SINAES. La metodología empleada fue un análisis descriptivo-analítico de los planes de mejora, basados en los componentes que propone la agencia para que las carreras realicen su proceso de autoevaluación, a saber: currículum, administración de la carrera o programa, estudiantes, impacto y pertinencia de la carrera o programa, personal académico, infraestructura y equipamiento. La información obtenida de cada componente se agrupó de acuerdo con las acciones de mejora, en categorías creadas por las investigadoras.

El análisis se dividió en dos fases: una, de carácter más descriptivo, donde se elaboraron las categorías de agrupación de las acciones de mejora de cada componente, que facilitara la clasificación de las necesidades prioritarias de las carreras. La construcción de las categorías de análisis consistió en tomar cada componente propuesto por la agencia y juntar las acciones más parecidas entre ellas $y$, posteriormente, darles un nombre que las englobara. Con las categorías de agrupación construidas se realizó un conteo en cada categoría del número de carreras con el fin de obtener la frecuencia.

La segunda fase, denominada analítica, consistió en la explicación de las tendencias globales de los datos, basándonos en las acciones planteadas por las carreras dentro de las necesidades de mejora de la institución Adicionalmente, se conceptualizó el significado de las categorías de análisis, lo que permitió detectar grandes temas institucionales o patrones en los datos más frecuentes. 


\section{Principales hallazgos}

\subsection{Descripción del área de pertenencia de las carreras acreditadas}

En este estudio se tomaron los dieciséis planes de mejora presentados por las carreras acreditadas de la Universidad de Costa Rica del 2001 hasta el mes de julio de 2010.

Si ubicamos las 16 carreras acreditadas según las áreas de conocimiento de la Universidad de Costa Rica se puede apreciar que seis carreras pertenecen al área de la Salud y seis pertenecen al área de Ciencias Sociales, mientras que las áreas Agroalimentarias, Ciencias Básicas, Ingeniería y Artes y Letras, tienen únicamente una sola carrera acreditada por el SINAES (Ver Gráfico No. 1).

En el eje vertical del Gráfico No. 1, se muestran los años en que las carreras de estudio obtuvieron el certificado de acreditación por parte de la agencia, de modo que en la Universidad de Costa Rica la primera carrera se acreditó en el año 2001. Posteriormente, las siguientes carreras se acreditaron en un promedio aproximado de dos por año.

Gráfico No. 1.

Número de carreras acreditadas por año según área de estudio.

Universidad de Costa Rica.

2001-2010

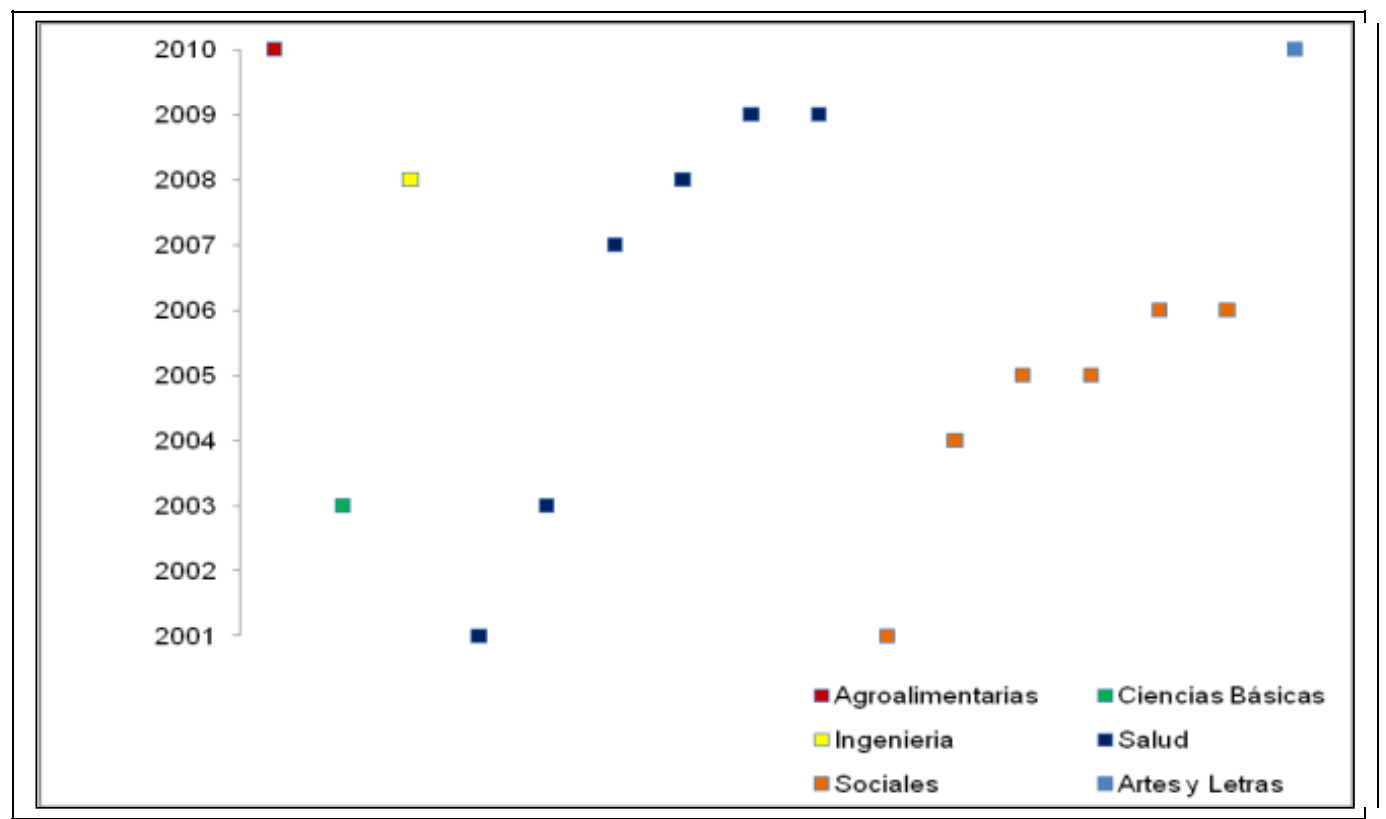




\subsection{Aspectos priorizados por las carreras en el componente personal académico}

Según el Manual de Acreditación del SINAES, la carrera debe contar con personal académico idóneo y suficiente para: garantizar la calidad de la docencia, la participación en la gestión curricular y en actividades de investigación y desarrollo profesional, para ello, debe realizar acciones específicas para mejorar la calidad profesional y académica de su personal docente (SINAES, 2000, p. 6).

A partir de la información revisada en los dieciséis compromisos de mejora, en este componente las acciones expresadas por las carreras se agruparon en nueve categorías de análisis, a saber: 1. Formación y reclutamiento del personal docente, 2. Tiempo docente, 3. Condiciones laborales, 4. Planificación de la docencia, 5. Producción y participación docente, 6. Mecanismos de evaluación, 8. Reglamentos y Normativa Universitaria y 9. Otro. Estas categorías esta definidas en los siguientes párrafos.

Las acciones agrupadas en la categoría denominada Formación y reclutamiento del personal docente, se refieren al reclutamiento del personal académico con el fin de reemplazar a docentes que se pensionan y a la necesidad de formación del personal docente desde diferentes aristas. También, aquí se encuentran consideraciones relacionadas con la existencia de docentes jóvenes con poca experiencia y poco dominio de las áreas básicas de la disciplina, actualización de los docentes tanto a nivel nacional como internacional, capacitación en docencia (favoreciendo el uso de las técnicas, las nuevas tecnologías, las competencias pedagógicas), así como la capacitación de los docentes para ejercer sus funciones como profesor y/o profesora consejera y la necesidad de posgrados en la disciplina. Para este componente, 15 carreras $(93,75 \%)$, plantean esta categoría como una acción de mejora dentro de sus planes (Ver Gráfico No. 2).

La segunda categoría encontrada fue el Tiempo docente, la cual comprende acciones de mejora dirigidas a los tiempos de contratación de los docentes, con el fin de reducir la falta de tiempos que se requieren para la atención de estudiantes, y para la atención del componente práctico de la carrera. Asimismo, se señala la falta de tiempos tanto para la investigación como para los trabajos finales de graduación de la población estudiantil de la carrera, así como la falta de tiempos para proyectos de acción social. En consecuencia, catorce carreras $(87,50 \%)$ proponen acciones de mejoras relacionadas con esta categoría (Ver Gráfico No. 2). 
En la categoría Condiciones laborales se agrupan acciones tendientes a mejorar las condiciones laborales como el interinazgo, sobrecarga de trabajo y mejorar los ingresos del personal docente. En esta categoría 13 carreras (81,25\%) proponen acciones (Ver Gráfico No. 2).

En la categoría Distribución y planificación de la docencia, se tomaron en cuenta acciones tales como la carencia de planes estratégicos donde se incorporen actividades de capacitación y de desarrollo docente, la inclusión de los mecanismos de supervisión del personal docente y los planes de investigación. También, se incluyen acciones tendientes a favorecer el establecimiento de un perfil de competencias acompañado de un programa de inserción de docentes nuevos. Por último, se incluye la necesidad de una adecuada distribución del tiempo docente, así como mejorar los mecanismos de comunicación. En estas dos últimas acciones la mitad (50,00\%) de las carreras coincidieron en incorporar acción de esta categoría en sus planes de mejora (Ver Gráfico No. 2).

Al igual que la categoría anterior, la mitad (50\%) de las carreras coincidieron en incorporar acciones en sus planes de mejoras relacionadas con la Producción $\boldsymbol{y}$ participación docente (Ver Gráfico No. 2). Esta categoría se refiere a la baja producción de los docentes en el ámbito de la investigación, desarrollo profesional y acción social, así como la baja participación de los docentes que realizan prácticas fuera de la universidad.

Por último, las acciones de mejora que se agruparon en la categoría Evaluación del personal docente se relacionan con la evaluación del desempeño docente, la evaluación de cursos y de los servicios de consejería y tutoría y la de Reglamentos y normativa universitaria, cuyas acciones de mejora están dirigidas al conocimiento de los Reglamentos y Normativa Universitaria especialmente en lo que se refiere al Reglamento de Régimen Académico Estudiantil. En la primera de ellas, 5 carreras (31.25\%) mencionaron acciones de mejora, y para la segunda 3 carreras consideran acciones relacionadas con esta categoría.

La categoría Otro consideró aquellas acciones de mejora que se encontraron en el componente de personal académico, pero no fueron ubicadas en ninguna de las nueve categorías mencionadas (Ver Gráfico No. 2). 
Gráfico No. 2

Componente Personal Académico por categorías de análisis según número de carreras acreditadas Universidad de Costa Rica.

2001-2010

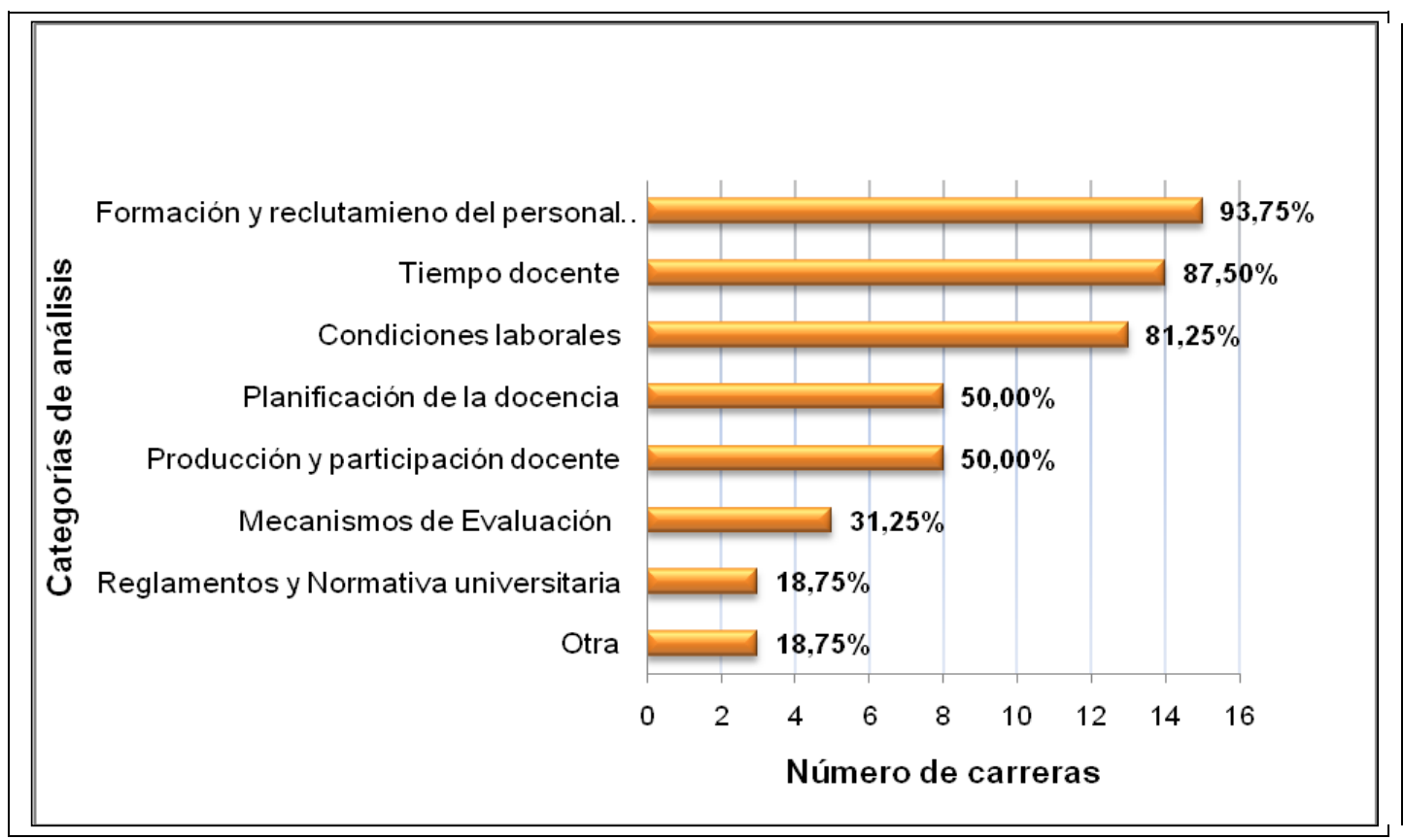

\subsection{Aspectos priorizados por las carreras en el componente currículum}

De acuerdo con el Manual de acreditación de la agencia SINAES (2000), el currículum debe hacer explícitas las intenciones educativas generales de la carrera o programa (metas, objetivos, misiones) y servir de guía al docente en la ejecución del currículum. Además, la carrera o programa debe tener un buen diseño, controlar su ejecución y evaluar los resultados (SINAES, 2000; p. 7).

En este componente se construyeron ocho categorías de análisis, o maneras en que se ha organizado la información contenida en los planes de mejora estudiados, estos son: 1. Actualización curricular, 2. Perfil profesional, 3. Evaluación, 4. Mejoramiento didáctico, 5. Conformación de comisiones de trabajo, 6. Incorporación de nuevas áreas al plan de estudio, 7. Reducción del índice de deserción y 8. Reducción de tiempo de graduación.

Como su nombre lo indica, dentro de la categoría Actualización curricular, las carreras incluyen aquellas acciones de mejora que buscan actualizaciones curriculares, mejoras en el plan de estudio, así como algunos aspectos específicos para incorporar concretamente en los cursos de la carrera. Dentro de las acciones concretas para llevar a 
cabo una revisión integral del plan de estudio y cambios curriculares, las carreras planean actualizar el plan de estudio de manera tal que éste responda a las demandas sociales, políticas y económicas del país, también proponen revisar la secuencia de cursos y de requisitos, revisar la fundamentación y la orientación de la carrera, evaluar la congruencia curricular, revisión del currículum para responder mejor a las necesidades del Ministerio de Educación Pública (MEP) y al mercado laboral, flexibilizar la propuesta curricular, y revisar los objetivos de la carrera y el grado de cumplimiento de los mismos, entre otros aspectos.

Destaca que 15 de las 16 carreras plantean, en sus planes de mejora, acciones que se clasificaron bajo esta categoría, lo que corresponde a un 93.75\% (Gráfico No. 3); aquí también se mencionan acciones concretas para mejorar los cursos, tales como: mejorar la coherencia entre cursos teóricos y prácticos, mejorar la coherencia entre el plan de estudios y la práctica profesional, buscar una mayor organización, sistematización y profundidad en las prácticas, mejorar la relevancia de los cursos y revisar los contenidos de los programas de los cursos.

La segunda categoría se refiere al estado y la Actualización del perfil profesional de la carrera; concretamente, las carreras encontraron que su perfil profesional debe revisarse y evaluarse para que esté acorde con la realidad nacional. Esta categoría es mencionada por 9 carreras $(56.25 \%)$.

En tercer lugar, se ubican las acciones relacionadas con la Evaluación y con el diseño de mecanismos para evaluar diversos rubros de interés para las carreras, tales como: evaluar el proceso de enseñanza-aprendizaje, el plan de estudio, los programas de los cursos, la práctica docente, la congruencia vertical y horizontal del plan de estudio, la función del director y el personal docente. Esta categoría es mencionada por 6 carreras (37.50\%).

Posteriormente, se encuentran las acciones que buscan el Mejoramiento didáctico y, en este sentido, la opción que más comúnmente se menciona es el incremento de la aplicación multimedia en la estrategia didáctica utilizada en la carrera. Esta categoría es mencionada por 5 carreras (31.25\%).

La quinta categoría de análisis se refiere al papel que pueden desempeñar las Comisiones de trabajo en tareas concretas que se relacionan con el componente currículum. Es así como las carreras acreditadas proponen crear comisiones para realizar funciones que van desde la actualización del plan de estudio, velar por la calidad de la enseñanza, discutir y aprobar programas de cursos, fortalecer la coordinación por 
departamentos, hasta el diseño de un sistema para determinar el cumplimiento de los objetivos de la carrera, entre otras funciones (Ver Gráfico No. 3). Esta categoría es mencionada por 5 carreras (31.25\%).

En la categoría denominada Incorporación de nuevas áreas al plan de estudio, se incluyen las acciones de mejora tendientes a incorporar en el plan de estudio una serie de áreas que las carreras consideran importantes en la formación del estudiantado y que están ausentes dentro del plan de estudio, o son débiles en el momento de la acreditación. Al mismo tiempo, se incluyen las acciones que pretenden robustecer la dimensión internacional de la carrera y enriquecer los vínculos externos e internos, que fortalezcan el plan de estudio. Dentro de los aspectos citados para fortalecer el plan de estudio las carreras consideran importante el área de negocios, el enfoque empresarial, el aprendizaje de idiomas, el aprendizaje de tecnología, el reforzamiento del aprendizaje del idioma inglés, mejorar las competencias en investigación de los y las estudiantes y mejorar los conocimientos que tiene el estudiantado sobre la realidad nacional (Ver Gráfico No. 3). Esta categoría es mencionada por 5 carreras (31.25\%).

Finalmente, la duración de los Tiempos de graduación y los índices de deserción de la carrera preocupan a algunas de las carreras acreditadas, por lo que se proponen reducir los tiempos y bajar los índices. Esta categoría fue mencionada por dos carreras (12.50\%). (Ver Gráfico No. 3).

Gráfico No. 3

Componente Currículum por categorías de análisis según número de carreras acreditadas Universidad de Costa Rica. 2001-2010

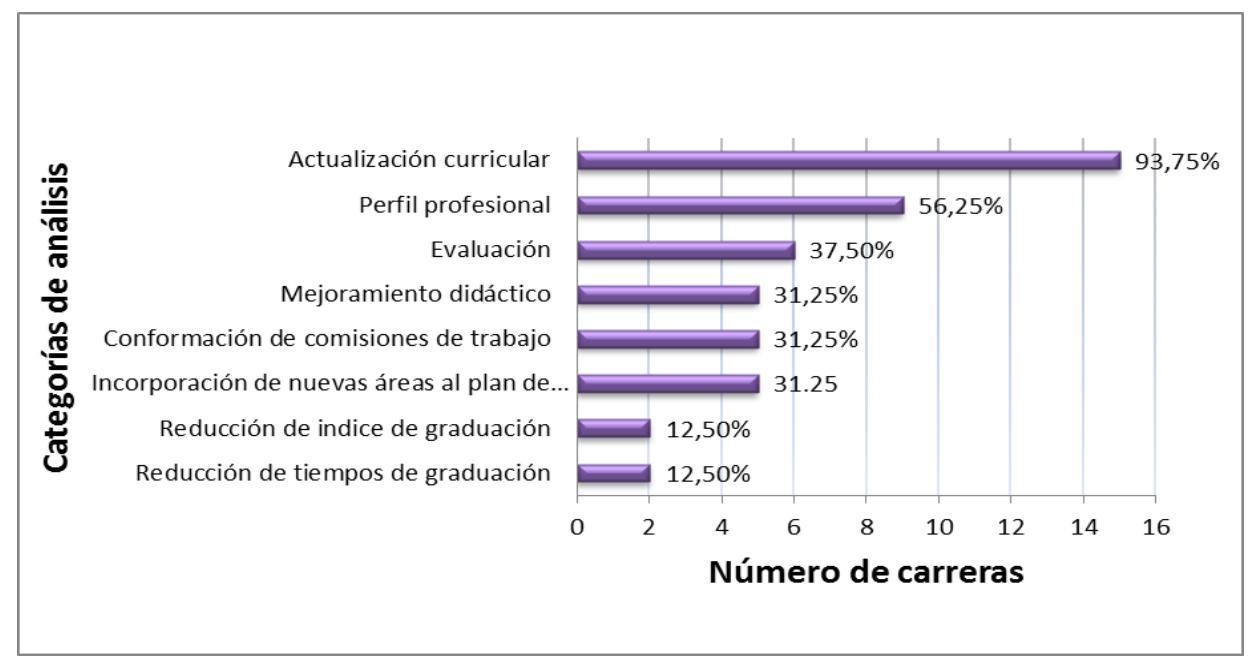




\subsection{Aspectos priorizados por las carreras en el componente de estudiantes}

De acuerdo con el Manual de acreditación de la agencia SINAES, para el componente Estudiantes es importante contar con políticas y estrategias para la conformación de la población estudiantil y ofrecerle condiciones y facilidades que favorezcan su permanencia como estudiante y el éxito en sus estudios (SINAES 2000, p. 9).

En este componente se construyeron 6 categorías de análisis, a saber: 1 . Desconocimiento de reglamentos y normativa universitaria, 2. Fortalecer las relaciones de consejería académica, 3. Rendimiento académico, 4. Débiles mecanismos de difusión de la información, 5 Necesidades y servicios del estudiantado y 6. Propiciar espacios de participación. Las acciones prioritarias de las Unidades Académicas van orientadas a superar las siguientes debilidades.

En la categoría denominada Desconocimiento de reglamentos y normativa universitaria, se refieren las carreras a la necesidad de mejorar la condición del estudiantado que desconoce la información sobre reglamentos y normativa que legislan el quehacer universitario, así como los derechos y las obligaciones que le demanda la legislación. En esta categoría se apuntan como evidencias el desconocimiento del reglamento de régimen académico estudiantil, las políticas de bienestar estudiantil, los procesos administrativos, el plan de estudio, sus cursos y costos entre otros. Para este componente, 12 carreras (75\%), plantean esta categoría como una acción de mejora (Ver Gráfico No. 4).

Seguidamente se expresa el interés prioritario de fortalecer las relaciones de consejería académica. Esta categoría se refiere a acciones para asegurar que el estudiantado reciba asesoría y atención académico-curricular apropiada y oportuna. Aclarar el papel del profesor consejero, vigilar que la relación sea eficiente, permanente y con variedad de horarios, asimismo, que el profesor o profesora consejera se preocupe por el rendimiento académico del estudiantado y su promoción rápida. Para este componente, 10 carreras (62.5\%) plantean esta categoría como acción de mejora (Ver Gráfico No. 4).

Relacionado con esta preocupación, la mitad de las carreras (50\%) señalan acciones relacionadas con el Rendimiento académico. Esta categoría recoge la preocupación de dar respuesta a aspectos como el alto número de ingreso de estudiantes, en el sentido de que el docente se enfrenta a grupos muy numerosos y, quizás, eso pueda influir negativamente en el porcentaje de aprobación de sus estudiantes, la condición especial o condición académica 
de alerta es de un $20 \%$ del estudiantado. Esta condición especial se aplica cuando un estudiante obtiene un promedio ponderado inferior a siete durante un año lectivo. También, se incluyó la falta de bases académicas para llevar con éxito cursos de servicio y deficiencias en el manejo de las herramientas básicas de informática (Ver Gráfico No. 4).

Tal como queda consignado en el párrafo anterior, se vislumbra la preocupación de las carreras acreditadas de informar al estudiantado, apropiadamente, no sólo de la reglamentación universitaria, sino de todos los servicios y actividades disponibles que ofrece la carrera, ya que se ha evidenciado que no es siempre oportuna y completa. Esta categoría denominada Débiles Mecanismos de difusión y de información es prioritaria para el 50\% de las carreras acreditadas (Ver Gráfico No. 4).

La categoría Necesidades y servicios del estudiantado agrupa necesidades específicas expresadas por el sector estudiantil, como fotocopiadoras, una cafetería, servicios de Orientación vocacional y una evaluación a la prueba de habilidades que algunas carreras solicitan como requisito de entrada. Para este componente, 7 carreras (43.75\%) plantean esta categoría como una acción de mejora (Ver Gráfico No. 4).

Por último, en un menor porcentaje con 18.75\% (Ver Gráfico No. 4), la categoría Propiciar espacios de participación evidencia la necesidad del estudiantado para expresar sus opiniones en instancias.

Gráfico No. 4

Componente Estudiantes por categorías de análisis según número de carreras acreditadas Universidad de Costa Rica. 2001-2010

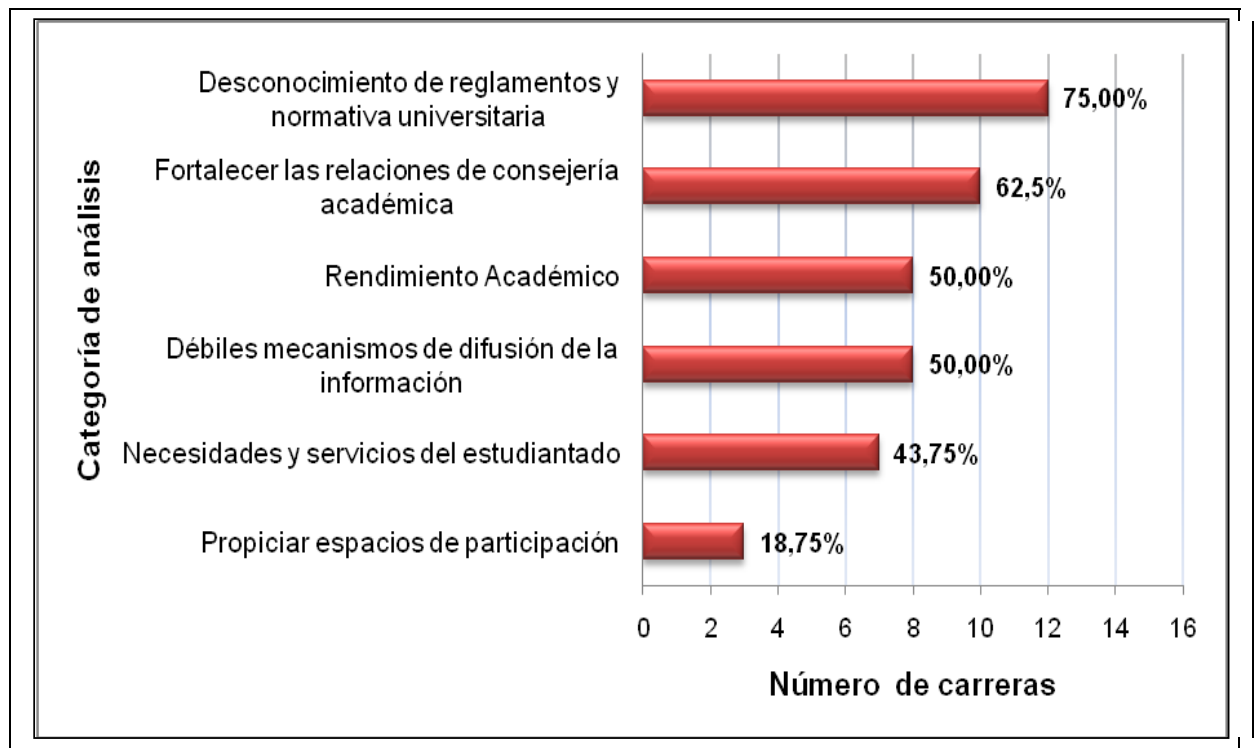




\subsection{Aspectos priorizados por las carreras en el componente administración de la carrera}

De acuerdo con el Manual de acreditación de la agencia SINAES convocatoria 2000, la administración de la carrera debe garantizar la existencia de sistemas de apoyo para la realización de las labores académicas, que favorezcan la comunicación interinstitucional, promuevan la superación del personal y la formación integral de los estudiantes (p. 11).

A partir de la información contenida en los planes de mejora, en este componente se construyeron 9 categorías de análisis, a saber: 1. Bases de datos, 2. Comunicacióndivulgación, 3. Recursos financieros, 4. Población estudiantil, 5. Personal administrativotécnico, 6. Mejoras administrativas, 7. Personal docente, 8. Mejorar coordinación y condición de áreas física y 9 . Otra, que incluye todas aquellas acciones de mejora relacionadas con la infraestructura y no se encuentra en las categorías anteriores.

En primer lugar destaca que 13 carreras $(81.25 \%)$ decidieron que era prioritario contar con información organizada y clasificada en bases de datos, para que esta se convierta en un apoyo para la toma de decisiones de las autoridades. Es así como las carreras sugieren una amplia gama de bases de datos, dentro de las cuales se pueden mencionar la confección de registros en relación con la población activa de la carrera, sus características demográficas, seguimiento de admisión, permanencia, deserción, graduación, seguimiento a la población ya graduada, rendimiento académico en general y para control de la población estudiantil con bajo rendimiento académico, sus apelaciones y los programas de los cursos. También, se visualiza la necesidad de contar con bases de datos para temas relacionados con el personal docente, registros de sus capacitaciones, actualizaciones y perfeccionamientos. Asimismo, se propone sistematizar la información que se genera con el personal administrativo, utilizarla en la toma de decisiones y la mejora de la gestión académica desarrollando un plan estratégico (Ver Gráfico No. 5).

En segundo lugar, se encuentra la categoría de análisis llamada Comunicación y divulgación donde se incluyen acciones que buscan mejorar la comunicación con otras unidades académicas que brindan servicios y apoyo a la carrera, con las que se puede colaborar para fortalecer la investigación, el perfeccionamiento académico y fomentar la cooperación. Asimismo, se evidencian acciones tendientes a mejorar la comunicación con la administración universitaria, con la comunidad de estudiantes y docentes de la Escuela, tomando en cuenta las observaciones que hace el estudiantado. 
Dentro de esta categoría, también se visualizan acciones tendientes a fomentar la divulgación tanto a lo interno de la carrera como a lo externo de la universidad, dentro de lo que se incluye dar a conocer la misión, visión y valores de la carrera, las publicaciones en revistas indexadas del personal docente, en la página web, propiciar espacios de reflexión sobre el desarrollo de la carrera, de los informes internos realizados, del uso de mecanismos automatizados para divulgar aspectos de la carrera e imprimir información de la carrera para ponerla a disposición del público (Ver Gráfico No. 5). Esta categoría es mencionada por 9 carreras (56.25\%).

La mitad de las carreras acreditadas presentan en sus planes de mejora, acciones tendientes a mejorar la dotación de Recursos financieros de manera tal que se puedan atender con mayor eficacia las necesidades de los sectores académico, administrativo y estudiantil. Las acciones propuestas son: desarrollar una política de consecución de fondos externos, contar con garantía de inversión presupuestaria por parte de la universidad en el presupuesto ordinario, ya que la Universidad de Costa Rica cuenta con poco presupuesto para los gastos de operación, investigar, capacitación, acción social, adquisición de recursos didácticos, atender necesidades de docentes, infraestructura y materiales en general (Ver Gráfico No. 5).

Seguidamente, se encuentra la categoría denominada Población estudiantil en la que las carreras incluyen las acciones que procuran mejorar el servicio que se le presta al sector estudiantil, como por ejemplo, bajar el número de estudiantes por curso (apertura de más cursos), adecuar los horarios de los cursos, ofrecer tercer ciclo o cursos de verano, reducir el alto tiempo de permanencia de la población estudiantil en la carrera, estableciendo metas de tiempo de permanencia y estableciendo un plan de estudio realista para el tiempo en que tienen los y las estudiantes para graduarse. Otro aspecto que se desea es buscar procedimientos ágiles para la atención de estudiantes, incentivar la participación de la población estudiantil en asambleas de escuela e incorporar estudiantes en la gestión de la carrera (Ver Gráfico No. 5). Esta categoría es mencionada por 8 carreras (50\%).

En la categoría Personal administrativo-técnico se agrupan las acciones de mejora relacionadas con el personal administrativo y técnico que colabora en la carrera, donde destaca la insuficiencia de personal y la importancia de evaluar y capacitar a esos sectores (Ver Gráfico No. 5). Esta categoría es mencionada por 7 carreras (43.75\%). 
Seguidamente, en la categoría mejoras administrativas las carreras incluyen aquellas acciones de mejora que se enfocan en el fortalecimiento de los servicios administrativos, lo cual implica agilizar los procedimientos para la atención de estudiantes, el desarrollo de evaluaciones a los servicios académicos y administrativos que se prestan, las mejoras en la compra, distribución y utilización de los recursos y la reorganización de las funciones de los sectores administrativos, técnicos y docentes. Además, se mencionan evaluaciones a los servicios administrativos, evaluación a la calidad de la carrera y de los cursos (Ver Gráfico No. 5). Esta categoría es mencionada por 7 carreras (43.75\%).

En la categoría Personal docente 5 carreras (31.25\%) señalan la necesidad de incentivos no salariales para este sector, así como la importancia de evaluar la gestión académica y la creación de mecanismos de evaluación. También, se muestran acciones para el mejoramiento de la calidad y el control de la gestión académica- administrativa, entendida ésta como el logro de las acciones bajo procedimientos claros para todos los actores, desarrollar un perfil para seleccionar personal docente, ejecutar los resultados obtenidos de la evaluación académica (Ver Gráfico No. 5).

Finalmente, se encuentran las acciones tendientes a mejorar la Coordinación/condición de las áreas físicas, donde se proponen una serie de acciones para mejorar laboratorios, centros de cómputo para estudiantes y espacios físicos de los que dispone el personal docente (Ver Gráfico No. 5). Esta categoría es mencionada por 2 carreras $(12.50 \%)$.

Gráfico No. 5

Componente Administración de la carrera por categorías de análisis según número de carreras acreditadas Universidad de Costa Rica.

2001-2010

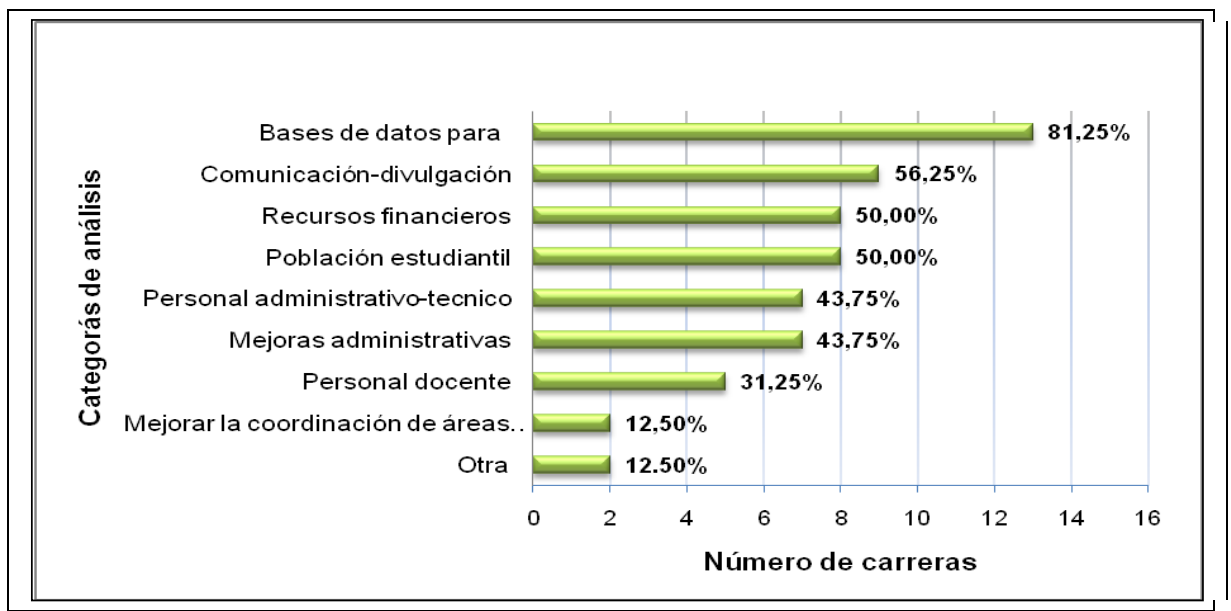




\subsection{Aspectos priorizados por las carreras en el componente infraestructura y equipamiento}

De acuerdo con el Manual del SINAES, la carrera o programa debe contar con los recursos físicos y materiales necesarios en cantidad, calidad, pertinencia, disponibilidad y acceso, para el cumplimiento de sus propósitos y objetivos. Asimismo, debe prever en sus planes de desarrollo y en sus proyecciones de crecimiento académico, la adquisición, el mantenimiento y la reposición de estos recursos (SINAES 2000, pág 11).

En este componente se construyeron nueve categorías de análisis, a saber: 1 . Mejora de la planta física existente, 2. Recursos bibliográficos, de computación y multimedios, 3. Instalaciones para la docencia, 4. Espacio de atención de estudiantes y reuniones, 5 Equipo de la carrera, 6. Accesibilidad arquitectónica, 7. Normas de seguridad, 8. Administración de los recursos e infraestructura y 9 . Otra

Las dieciséis carreras acreditadas (100\%) han expresado en sus planes de mejora acciones dirigidas a Mejorar la planta física existente, entre ellas se pretenden optimizar la distribución del espacio físico, realizar mejoras en las instalaciones físicas para las actividades administrativas de la carrera y la falta de aulas. Asimismo, se plantea mejorar el mantenimiento general del edificio en lo que refiere a los servicios sanitarios de la planta física utilizada por la carrera, así como la rotulación, iluminación y la limpieza en general (Ver Gráfico No. 6).

En una segunda categoría, catorce $(87,5 \%)$ de las carreras mencionaron incorporar dentro del plan de mejora acciones relacionadas con la categoría Recursos bibliográficos, de computación y multimedios. En esta categoría se encuentran acciones relacionadas con la adquisición de mayor cantidad y actualización de los libros de texto, bibliotecas especializadas, más suscripciones a revistas, accesos a redes de información y mecanismos de verificación de las necesidades de libros especializados. En cuanto al equipo de computación y multimedia las acciones van dirigidas al aumento del personal administrativo para su mantenimiento, aumentar la cantidad de equipo de cómputo, equipo multimedia, ofrecer disponibilidad del equipo de cómputo en horarios nocturnos. Al mismo tiempo, se pueden encontrar aspectos relacionados con la capacitación en el uso de paquetes computacionales, uso de material audiovisual y difusión o creación de una página web (Ver Gráfico No. 6). 
En la categoría Instalaciones para la docencia diez carreras (62.5\%) incluyeron en su plan aquellas acciones relacionadas con el espacio físico necesario para que el cuerpo docente realice sus labores, como por ejemplo, la falta de aulas especializadas, laboratorios y otros lugares que favorezcan las actividades de aprendizaje de los y las estudiantes. Además, se incluye la necesidad de salas de cómputo (Ver Gráfico No. 6).

Siete carreras (43.75\%) ubicaron acciones de mejora en la categoría denominada Espacio de atención de estudiantes y reuniones. En esta categoría se agrupan las necesidades de mejora de la infraestructura para la atención de estudiantes por parte de los y las estudiantes, reuniones con el cuerpo docente y para sus presentaciones (Ver Gráfico No. 6).

Dentro de la categoría Accesibilidad arquitectónica seis de las unidades académicas acreditadas (37.50\%) plantearon en sus planes dotar a los edificios de distribución para las personas con algún tipo de discapacidad física. El mismo número de carreras plantearon sus acciones en la categoría Equipo de la carrera, que está relacionada con el mantenimiento del equipo de cómputo, actualización del equipo especializado de la carrera y mayor cantidad de recursos multimedios que faciliten el desarrollo de las actividades académicas (Ver Gráfico No. 6).

En la categoría Normas de seguridad 5 carreras (31.25\%) propusieron acciones relacionadas con la misma. Esta categoría se compone de la incorporación de salidas de emergencia de la planta física, mayor regulación de las normas de seguridad, higiene ambiental y salud ocupacional. Mejorar los protocolos de salud y seguridad, mayor control de la contaminación sónica. Mejorar la seguridad del edificio de los robos en los edificios y alrededores (Ver Gráfico No. 6).

Por último, en dos carreras (12.5\%), se mencionan acciones contenidas en la categoría Administración de los recursos e infraestructura. Aquí se plantean acciones dirigidas a la planificación de las necesidades de infraestructura y equipo, crear planes de desarrollo donde se estipule la adquisición, el mantenimiento y la reposición de recursos. Establecer las necesidades de los estudiantes con respecto al equipo multimedio (Ver Gráfico No. 6). 


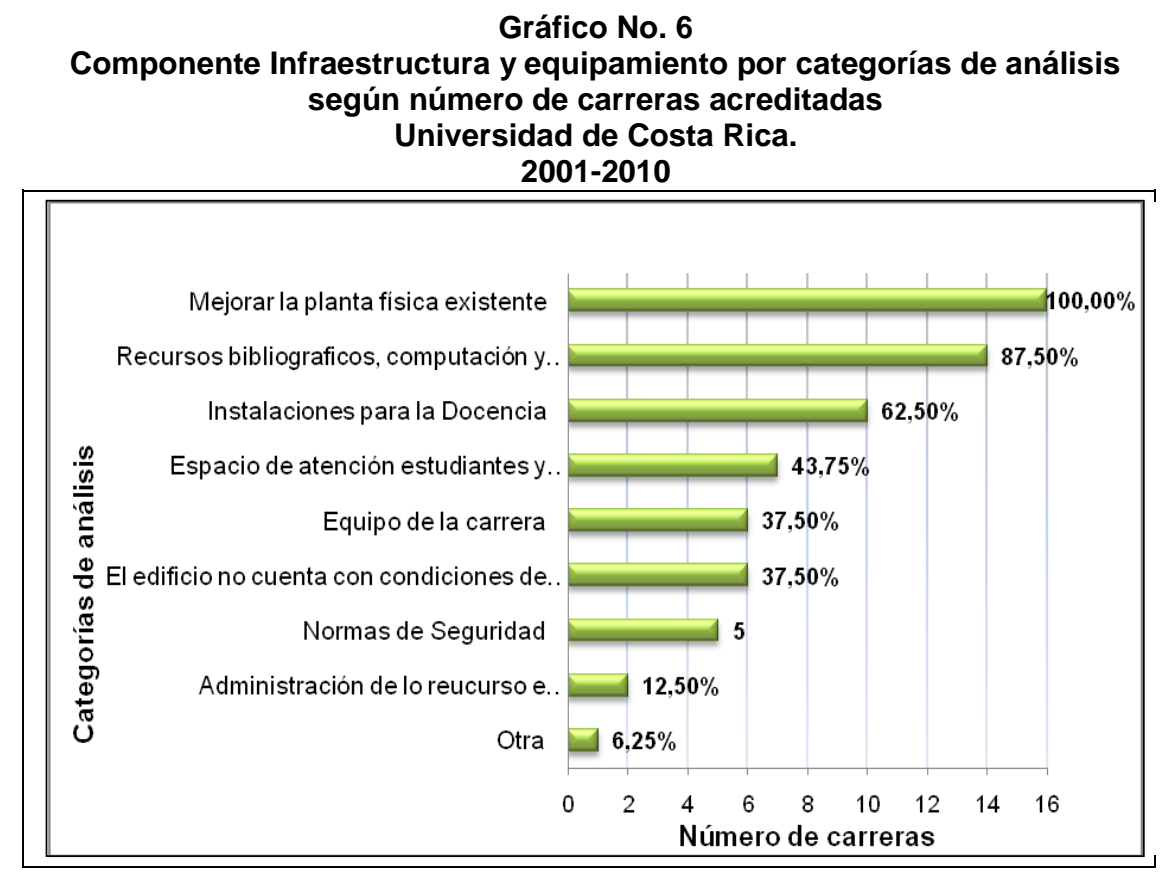

\subsection{Aspectos priorizados por las carreras en el componente de impacto y pertinencia}

De acuerdo con el Manual de acreditación de la agencia SINAES, en este componente de impacto y pertinencia, la carrera debe promover eventos académicos que enriquezcan la formación de los estudiantes y profesores, hacer aportes y promover transformaciones en la comunidad en que está inmersa y realimentar su quehacer en forma sistemática (SINAES, 2000, p. 13).

A partir de la información revisada en los dieciséis compromisos de mejora, en este componente las acciones expresadas por las carreras se agruparon en cinco categorías de análisis: 1. Vinculación académica, 2. Comunicación y divulgación, 3. Actividades académicas extracurriculares, 4. Fortalecer la investigación y la evaluación, 5. Propiciar la capacitación.

Las acciones agrupadas en la categoría denominada Vinculación académica se refieren a fortalecer la carrera en una serie de actividades orientadas a vincular al personal docente como al estudiantado, con universidades e institutos de investigación nacionales y extranjeros. También, se intentan promover relaciones de intercambio permanente con otras unidades académicas, empleadores, egresados, colegio profesional y centros de 
investigación afines. Otros aspectos que se mencionan consisten en vincular la actividad investigativa de los institutos con la labor docente y la acción social, la falta de estrategias para ubicar y fortalecer la carrera en el ámbito institucional, nacional e internacional y la importancia de consolidar un sistema de intercambio y convenios con universidades nacionales y extranjeras. Otra de las acciones apuntadas en esta categoría es establecer un sistema de venta de servicios. Para este componente 10 carreras (62.5\%) plantean esta categoría como una acción de mejora (Ver Gráfico No. 7).

La segunda categoría encontrada fue Comunicación y divulgación, la cual comprende acciones dirigidas a la proyección que realiza la unidad académica con la sociedad por medio de publicaciones en libros y revistas científicas y profesionales, así como la difusión de información y actividades a la comunidad académica. Dentro de las acciones señaladas se encuentran las siguientes; difundir la producción intelectual y profesional de la escuela, mejorar la difusión de las actividades, optimizar los canales de comunicación con los estudiantes, mejorar el débil protagonismo en la resolución de los problemas nacionales, mejorar la escasa proyección a la comunidad y propiciar un sistema de intercambio con el sector empleador. Para este componente 8 carreras (50\%) plantean esta categoría como una acción de mejora (Ver Gráfico No. 7).

Al igual que la categoría anterior, la mitad (50\%) de las carreras coincidieron en incorporar acciones en sus planes de mejora relacionadas con la categoría Actividades académicas extracurriculares. En esta categoría se agrupan acciones tendientes a promover la participación de la comunidad costarricense en general y del estudiantado en particular de eventos académicos auspiciados y generados por la unidad académica como conferencias, seminarios, charlas, debates, foros (Ver Gráfico No. 7).

En la categoría de Investigación y evaluación se mencionan inquietudes específicas de algunas carreras acreditadas, que plantean investigar o evaluar diversos rubros de interés, para dar respuesta a ciertos fenómenos propios de la carrera. En 6 carreras (37.5\%) plantean esta categoría como una acción de mejora (Ver Gráfico No. 7). Estos estudios particulares son: 1. Retroalimentar por parte de empleadores y graduados la profesional, 2. Estudiar la inserción laboral de estudiantes y la situación del mercado laboral, 3. Promover otro nombre o título a la carrera porque se confunde con otros programas, 4 . Crear un centro de investigación. 
Por último, en la categoría Propiciar la capacitación, se incluyen acciones relacionadas con la escasa oportunidad que tiene el personal docente y estudiantil de las unidades académicas acreditadas, de participar en pasantías e intercambios, y de conocer las necesidades de capacitación de los egresados. Para este componente 4 carreras (25\%) plantean esta categoría como una acción de mejora (Ver Gráfico No. 7).

Gráfico No. 7

Componente Impacto y pertinencia por categorías de análisis según número de carreras acreditadas. Universidad de Costa Rica. 2001-2010

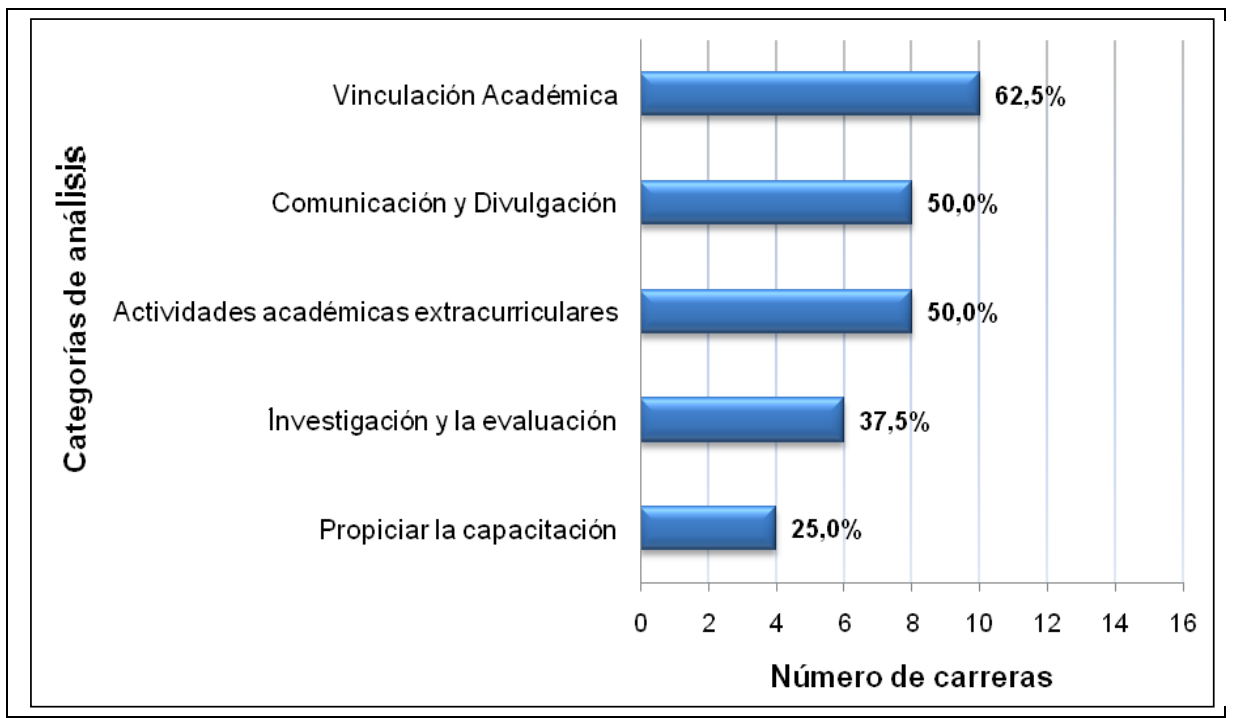

\subsection{Aspectos generales priorizados por las carreras en los planes de mejoramiento}

Al unificar las acciones de mejora planteadas en las 16 carreras analizadas, se encontró que las acciones con mayor frecuencia e importancia fueron las siguientes:

1. La primera que las dieciséis carreras expresaron la importancia de mejorar la planta física existente; en el apartado 3.6 se detallan las acciones que incluye esta categoría.

2. La segunda y la tercera acciones de mejora, que más mencionaron las carreras, fueron la de actualización curricular y la de formación y reclutamiento de personal docente con $15(93,75)$. Las acciones también se detallan en el apartado 3.3 y 3.2, respectivamente.

3. En cuarto y quinto lugar están las acciones de mejora referidas a las categorías de recursos bibliográficos, de computación y multimedio y tiempo docente, las cuales 
fueron mencionadas por 14 de las carreras (87,50\%), y están descritas en el apartado 3.6 y 3.3, cada uno (Ver Gráfico No. 8).

Gráfico No. 8

Principales categorías que priorizaron las carreras acreditadas de la Universidad de Costa Rica. 2001-2010

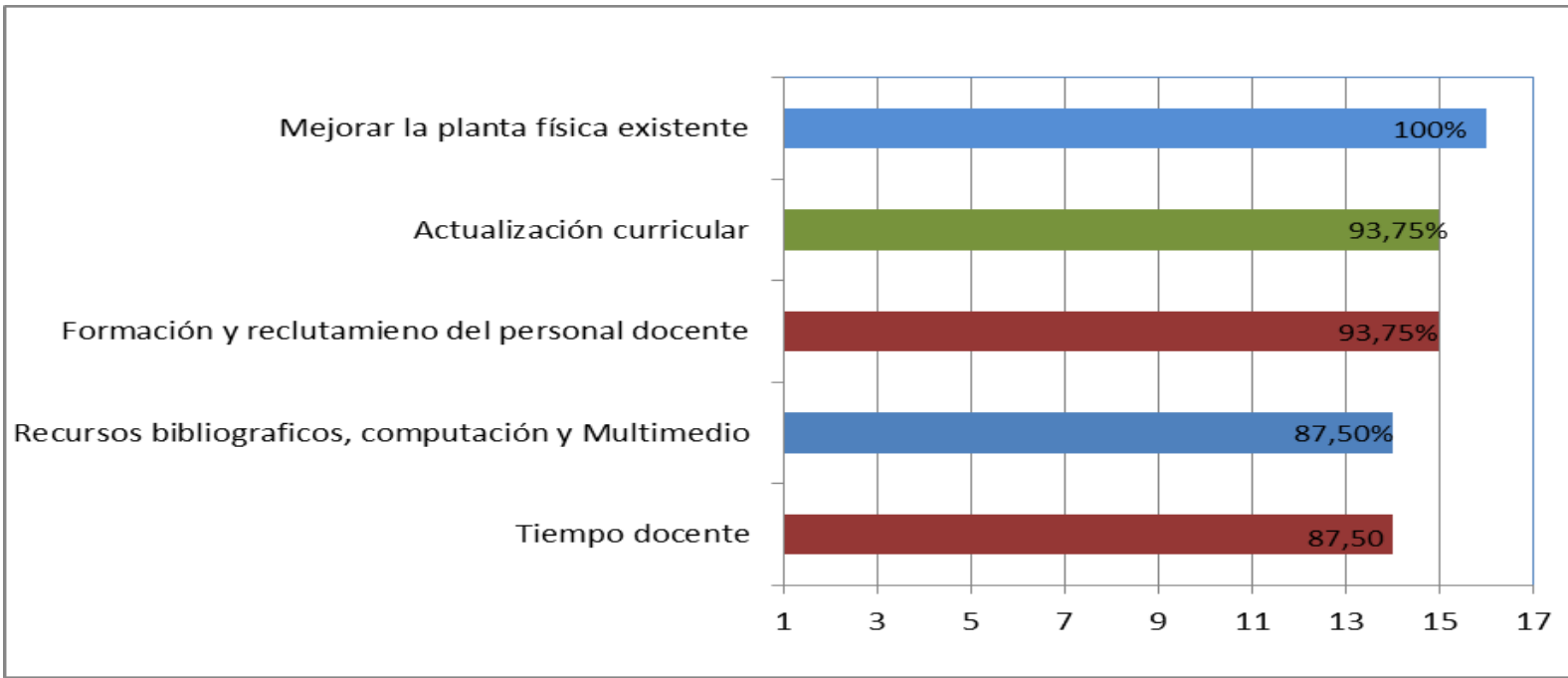

\section{Conclusiones}

Al analizar los planes de mejora presentados por las carreras acreditadas, es importarte diferenciar dos tipos de acciones encontradas: por un lado, aquellas acciones que dependen de la carrera propiamente, como son: la actualización curricular, las necesidades de las áreas disciplinares para formar su personal docente, la difusión de la normativa y, por otro lado, las acciones que dependen de instancias institucionales y, por ende, se salen del dominio de las carreras, ya que estas no pueden intervenir directamente en la acción, tales como las relacionadas con las mejoras de la planta física o la asignación de los tiempos docentes a las distintas carreras.

Asimismo, este estudio ha permitido evidenciar que, al momento de acreditarse, las carreras tienen debilidades o retos que superar mediante un planeamiento de todas aquellas acciones que deben ejecutarse, para alcanzar un mayor nivel de calidad. A pesar de esto, es posible que por su calidad se les acredite, ya que, evidentemente, la agencia considera que estas deficiencias son subsanables. 
No se puede deducir, a partir del análisis realizado, que las carreras acreditadas posean una cultura de la evaluación, tal como es definido por Bolseguí y Fuguet. Sin embargo, sí es evidente que la institución y las carreras acreditadas han venido implementando una serie de prácticas evaluativas y actividades de reflexión que tienden a alcanzar la denominada cultura de la evaluación planteada por los autores anteriormente citados. Algunas de las prácticas implementadas por las carreras son: la decisión de enfrentarse a procesos evaluativos participativos como la autoevaluación y la evaluación externa. Adicionalmente, elaboran una planificación para implantar acciones de mejoramiento, producto de procesos de reflexión de los actores involucrados en la carrera. Idealmente estas acciones tendrán un seguimiento para velar por su cumplimiento, lo que incentiva crear prácticas evaluativas periódicas, ya que las carreras, al parecer, han iniciado un proceso de poner en orden la casa.

Desde otro punto de vista, la institución dentro de sus políticas 2010-2014 ha establecido: "promover el desarrollo académico, institucional con base en procesos de monitoreo, evaluación, autoevaluación, autorregulación y acreditación" (Políticas Institucionales, 2010-2014).

Este estudio también ha permitido reflexionar acerca del concepto de calidad que promueve el SINAES en su modelo de acreditación. Según lo analizado, vale entonces preguntarse si la acreditación de una carrera se trata más bien de construir los basamentos de una carrera de calidad ya que pareciera ser que, el concepto de calidad planteado en términos de pasar a un estado mejor, solo podrá ser observado en una evaluación posterior a la puesta en marcha de los planes de mejora. Es entonces cuando se podría valorar en qué medida las acciones programadas en los planes de mejora inciden, de manera concreta y positiva, en los aspectos de calidad propuestos por la agencia y si el plan de mejora es, efectivamente, un listado de necesidades para garantizar esa calidad.

Aunque no es parte de este análisis, se puede vislumbrar que con los procesos de reacreditación que le siguen a las carreras acreditadas, se plantean una serie de retos a las carreras, a la agencia de acreditación y a la Universidad de Costa Rica. En este sentido, las carreras que optan por la reacreditación deberían consolidar el compromiso con la calidad académica asumido en la acreditación, además, superar las debilidades encontradas en el proceso anterior y fortalecer, de manera decidida, la cultura de evaluación por medio de la promoción de espacios de reflexión y profundización teórica en evaluación para fomentar 
procesos continuos, permanentes, contextualizados, flexibles, de naturaleza formativa y participativa.

Por último, tal y como lo plantea Rangel (2010, p.1) para que la evaluación propicie la adopción de una cultura evaluativa, es necesario que favorezca lo que él llama una "dinámica movilizadora de los actores", que sea participativa y rebase los procedimientos burocráticos que plantean a la evaluación como un proceso técnico-burocrático. En este sentido, se visualiza a la evaluación como un proceso donde las personas partícipes del proceso educativo reflexionen en conjunto, partiendo de la información que se genere en la autoevaluación.

\section{Referencias}

Bolseguí, Milagros \& Fuguet, Antonio. (2006). Cultura de evaluación: una aproximación conceptual. Revista Investigación y Posgrado, 21 (001), 77-98.

Costa Rica. (2002). Ley del sistema nacional de acreditación de la educación superior (SINAES) (Ley 8256, del 17 de mayo del 2002). San José: Asamblea Legislativa.

International Network for Quality Assurance Agencies in Higher Education. (2011). What can INQAAHE do for you?. Recuperado el 12 de julio 2011 de http://www.inqaahe.org/main/about-inqaahe/what-can-inqaahe-do-for-you

Sistema Nacional de Acreditación de la Educación Superior (SINAES). (2000). Manual de Acreditación. Convocatoria año 2000. San José, Costa Rica: El autor.

Sistema Nacional de Acreditación de la Educación Superior (SINAES). (2009). Manual de Acreditación Oficial de carreras de grado del Sistema Nacional de Acreditación de la Educación Superior. Recuperado el 30 de marzo de 2010, de http://www.sinaes.ac.cr/manual guias/manual oficial acreditacion vf feb2010.pdf

Rangel, Hugo. (2010). Hacia una evaluación generadora. Más allá de la evaluación técnico-burocrática de las universidades en México. Recuperado el 25 de marzo de 2010, de http://www.rieoei.org/deloslectores/3794Rangel.pdf

Teichler, Ulrich. (2007). ¿Qué es calidad?: una perspectiva desde Europa. En: Gilberto Alfaro et al. (eds.), Promover la universidad: cinco estrategias un dilema, la gestión de la calidad y el cambio en la educación superior (pp. 69-78). San José: EUNED.

Universidad de Costa Rica. (2001-2010). Planes de mejora de las carreras acreditadas por el SINAES. 